\title{
Automatic non-invasive differentiation of obstructive and central hypopneas with nasal airflow compared to esophageal pressure
}

\author{
C. Morgenstern, Member IEEE, M. Schwaibold, W. Randerath, A. Bolz and R. Jané, Member IEEE
}

\begin{abstract}
The differentiation of obstructive and central respiratory events is a major challenge in the diagnosis of sleep disordered breathing. Esophageal pressure (Pes) measurement is the gold-standard method to identify these events but its invasiveness deters its usage in clinical routine. Flattening patterns appear in the airflow signal during episodes of inspiratory flow limitation (IFL) and have been shown with invasive techniques to be useful to differentiate between central and obstructive hypopneas. In this study we present a new method for the automatic non-invasive differentiation of obstructive and central hypopneas solely with nasal airflow. An overall of 36 patients underwent full night polysomnography with systematic Pes recording and a total of 1069 hypopneas were manually scored by human experts to create a goldstandard annotation set. Features were automatically extracted from the nasal airflow signal to train and test our automatic classifier (Discriminant Analysis). Flattening patterns were non-invasively assessed in the airflow signal using spectral and time analysis. The automatic non-invasive classifier obtained a sensitivity of 0.71 and an accuracy of 0.69 , similar to the results obtained with a manual non-invasive classification algorithm. Hence, flattening airflow patterns seem promising for the noninvasive differentiation of obstructive and central hypopneas.
\end{abstract}

\section{INTRODUCTION}

$\mathrm{T}$ HE correct differentiation between obstructive and central hypopneas is of importance in Sleep Disordered Breathing (SDB) for the appropriate choice of treatment [1]. Esophageal pressure (Pes) measurement is the gold-standard technique for assessing respiratory effort and the identification of obstructive and central events [2, 3]. However, its invasiveness and complexity deters its usage in clinical routine. Therefore, researchers have been recently trying to develop non-invasive systems for the differentiation between central and obstructive apneas/hypopneas [4 - 7]. Still, these methods require technical procedures, e.g. forced oscillation technique, usually not acquired in clinical routine, limiting their widespread clinical adoption.

In this study we propose a new approach to non-invasively automatic differentiate central and obstructive hypopneas

Manuscript received April $1^{\text {st }}, 2010$. This work was supported in part by the Spanish Ministerio de Ciencia e Innovación under grant TEC2007-68076C02-01.

C. Morgenstern and R. Jané are with Institut de Bioenginyeria de Catalunya (IBEC), Dept. ESAII, Universitat Politècnica de Catalunya (UPC), and CIBER de Bioingeniería, Biomateriales y Nanomedicina (CIBER-BBN), Baldiri i Reixach 4, 08028, Barcelona, Spain (e-mail: cmorgenstern@ibec.pcb.ub.es, rjane@ibec.pcb.ub.es).

M. Schwaibold is with MCC-Med GmbH \& Co. KG, Karlsruhe, Germany W. Randerath is with Klinikum Bethanien, Solingen, Germany

A. Bolz is with Institute of Biomedical Engineernig, Karlsruhe Institute of Technology (KIT), Karlsruhe, Germany with the nasal airflow signal. Nasal airflow has been a popular clinical standard for a long time that is appropriate for hypopnea identification [2, 3]. A characteristic airflow pattern (flattening) can be observed in the airflow signal of inspirations with inspiratory flow limitation (IFL). IFL is usually caused by increased upper airway (UA) resistance that prevents the increase of airflow despite increasing respiratory effort [8, 9]. In a recent study [10], our group was able to prove that obstructive hypopneas present a significantly $(\mathrm{p}<0.01)$ higher incidence of IFL episodes than central hypopneas, as increased UA resistance is usually also the main cause of obstructive hypopneas [1]. That study [10] also showed that the IFL information contained in flattening patterns is critical for a successful non-invasive hypopnea differentiation. However, in that study [10] the IFL patterns were assessed invasively with the Pes/airflow - relationship, so the hypopnea classification was not entirely non-invasive. Nonetheless, our group recently demonstrated in a separate study [11] that IFL episodes can be assessed non-invasively by using the spectral and time content of an inspiration's airflow signal. Thus, by assessing IFL non-invasively with this methodology [11] and combining it with the hypopnea classification concept priorly outlined in [10], we should be able to obtain an automatic, entirely non-invasive hypopnea classification system solely based upon the airflow signal. The automatic system's scorings should be finally compared to manually obtained gold-standard (Pes) hypopnea annotations to ensure their clinical validity.

\section{Methodology}

\section{A. Subjects}

Thirty-six subjects had full nocturnal polysomnography (NPSG) with an 18-channel recorder (Somnolab sleep diagnosis system, Weinmann GmbH, Hamburg, Germany) at the sleep laboratories of Klinikum Bethanien hospital in Solingen, Germany. The clinical protocol was specifically designed and approved by the hospital's Ethics Committee. Thirty subjects were male and six were female. All subjects were lung-healthy without asthma nor COPD. Mean values $\pm \mathrm{SD}$ of the studied population for Apnea-Hypopnea Index (AHI) were $14.2 \pm 12.5$ events/h (range $1.08-91.2$ events/h) with a Hypopnea Index (HI) of $9.3 \pm 6.3$ events $/ \mathrm{h}$ (range $0.85-23.9$ events $/ \mathrm{h}$ ), body-mass-index (BMI) $28.9 \pm$ $4.6 \mathrm{~kg} / \mathrm{m} 2$ (range $21.0-41.9 \mathrm{~kg} / \mathrm{m} 2$ ) and age $52.2 \pm 15.7$ years (range 23 - 78 years). Given the complexity of Pes measurement, our 36 subjects represent a significant increase in comparison to other Pes studies [7 -8]. 


\section{B. Data acquisition}

To obtain the respiratory signal, a nasal cannula device (Weinmann $\mathrm{GmbH}$, Hamburg, Germany) was connected to a pressure transducer system (Weinmann GmbH, Hamburg, Germany). These systems are indicated by current NPSG guidelines [2, 3] for the identification of hypopneas and have been routinely used with the Pes signal for the identification and analysis of IFL $[8,9]$. The airflow signal was recorded with a sampling frequency of $32 \mathrm{~Hz}$ [3] and an 8-bit resolution. Esophageal pressure was recorded with an unidirectional pressure-tip catheter (UniTip catheter by UNISENSOR AG, Attikon, Switzerland) that consisted of a piezoresistive pressure sensor with an accuracy of $+/-0.6$ $\mathrm{mmHg}$, a sensitivity of $5 \mu \mathrm{V} / \mathrm{V} / \mathrm{mmHg}$ and a typical resolution of $[-100 \ldots+300 \mathrm{mmHg}]$. A separate pressure amplifier (ISOPRE-P amplifier, Standard instruments $\mathrm{GmbH}$, Karlsruhe, Germany) with a resolution of [-99 mmHg...+200mmHg] was connected to the catheter. The Pes signal was recorded with a sampling frequency of $16 \mathrm{~Hz}$ and a 12 bit resolution. The catheter was introduced through the patient's nostrils after spraying the nasopharynx with Xylocaine and positioned in the lower third of the esophagus [12].

Other physiological signals, like arterial oxygen saturation $\left(\mathrm{SpO}_{2}\right)$, body position, pulse, and thoracic and abdominal respiratory inductance plethysmography (ProTech, Services Inc, Mukilteo, WA, USA) were recorded with an 8-bit resolution. Also 2 electroencephalogram channels (C3A2/C4-A1), 2 electrooculogram (right/left), 1 submental electromyogram (EMG), 1 leg-EMG and an electrocardiographic (ECG) channel were systematically recorded with a sample frequency of $256 \mathrm{~Hz}$ and a 12-bit resolution, respectively.

\section{Event annotations}

Standard criteria [2] were applied to score sleep stages, apneas, hypopneas and other respiratory events. According to these criteria, a hypopnea lasts for at least 10 seconds and is identified by a clear decrease ( $>50 \%)$ from baseline in the amplitude of a valid measure of breathing during sleep or is associated with either an oxygen desaturation of $>3 \%$ or an arousal [2]. The baseline is defined as the mean amplitude of stable breathing and oxygenation in the two minutes preceding the onset of the event [2]. Following these criteria 1882 hypopneas were independently identified in the NPSG recordings of our thirty-six patients by a human expert blinded to the Pes signal (scorer A). For the scoring of a central apnea or a central hypopnea, a clear reduction in esophageal pressure swings from the baseline, as defined before, is required [2]. A second human expert (scorer B) reviewed the priorly manually identified hypopneas by scorer $\mathrm{A}$ and differentiated them into obstructive and central using the Pes signal. If a hypopnea's Pes signal did not allow a reliable differentiation, scorer $\mathrm{B}$ discarded the hypopnea. This could be the case due to swallowing or coughing artifacts and baseline drifts of the Pes signal related to body movements that impeded the reliable assessment of respiratory effort. Undifferentiated hypopneas, mixed hypopneas and apneas were excluded from this study. Thus,

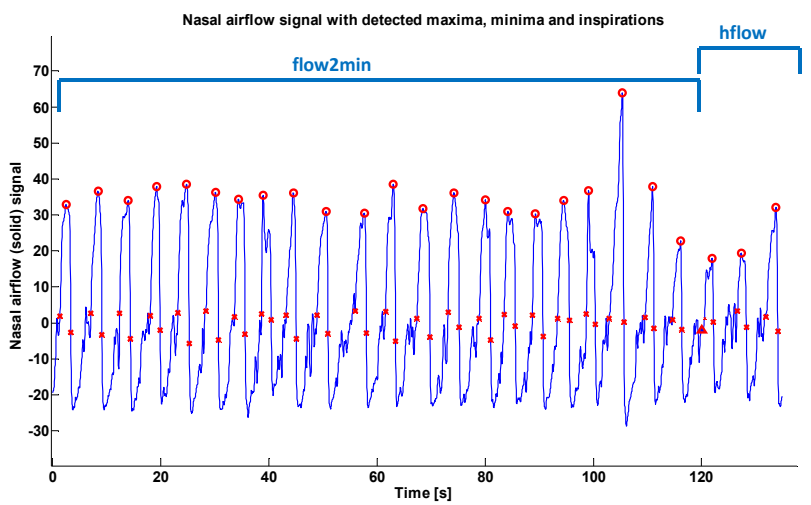

Fig. 1. Example of pre-processing for a central hypopnea. Maxima were detected (circles) for each respiratory cycle and the beginning and ending (crosses) of each inspiration. The manual hyopnea marker (triangle) indicates the beginning of the hypopnea. The signal was separated in a hypopnea segment (hflow) and into the 2 min prior to the hypopnea's onset (flow2min).

this resulted in an overall of 1069 manually scored, goldstandard hypopnea annotations, of which 611 were obstructive and 458 central hypopneas.

The number of discarded hypopneas due to the commented Pes artifacts reinforces the necessity for a non-invasive alternative for hypopnea differentiation. Nonetheless, the final amount of hypopnea annotations in this study represents a distinctive increase in manual, gold-standard (Pes) hypopnea annotations in comparison to other studies that e.g. only had an overall of 167 manually scored apneas and hypopneas [7].

\section{Pre-processing and detection of respiratory cycles}

As the automatic detection of hypopneas with classical PSG criteria [1] has already been proficiently solved [13], for the automatic processing the time markers of the 1069 manually identified hypopneas indicating the starting time and the duration in seconds of each hypopnea, were imported here.

The flow signal presented noise and physiological disturbances that had to be reduced. A low-pass filter with a cut-off frequency of $2.5 \mathrm{~Hz}$ is adequate for the analysis of the flattening airflow patterns when high-frequency oscillations (like snoring) are not to be analyzed [14]. Hence, a 5 point moving average (MA) low-pass filter with a cut-off frequency of $2.9 \mathrm{~Hz}$ at $-3 \mathrm{~dB}$ was applied to the flow signal. The MA filter was applied in forward and reverse directions in order to achieve zero-phase

The manual hypopnea time markers were used to separate the flow signal of the hypopnea (hflow) and the 2 min prior to the hypopnea's start (flow2min), see fig. 1. Respiratory periods in the extracted airflow signal were detected as described in [11] and the maxima and minima were obtained for each respiratory cycle, see fig. 1. Each inspiration was then individually extracted for separate processing. In the case that less than two inspirations could not be detected in a hypopnea due to artifacts, the whole hypopnea was discarded.

Following the methodology described in [11], five patients were randomly selected to create an IFL training set with an overall of 35,497 inspirations, of which 12,697 were IFL and 


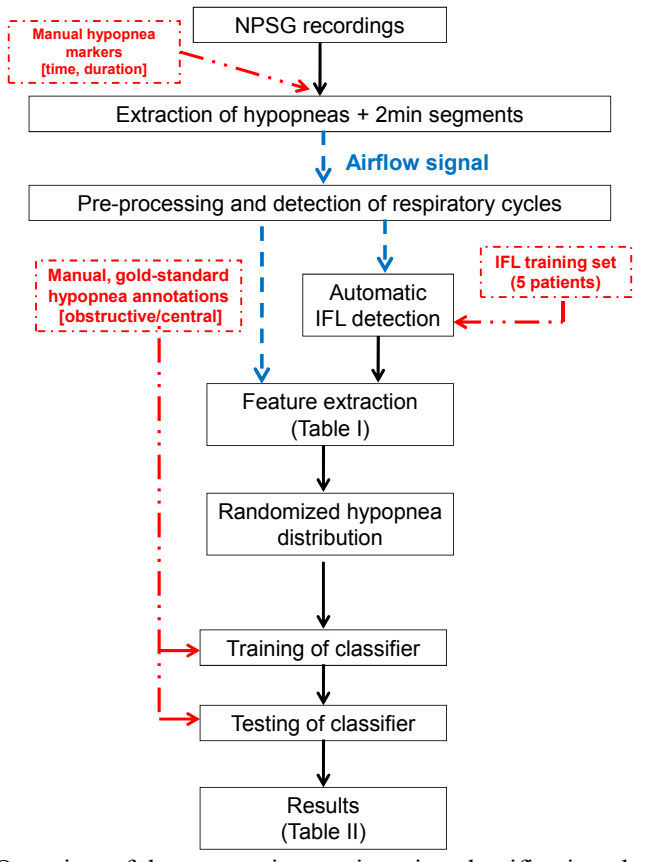

Fig. 2 Overview of the automatic, non-invasive classification algorithm

22,800 non-IFL inspirations according to prior obtained gold-standard (Pes) annotations [11]. The corresponding 99 hypopneas of these five patients were not used for the automatic hypopnea assessment. Of the remaining 970 hypopneas, five percent were discarded by our preprocessing algorithm because of artifacts in the Pes signal. Thus, a total of 918 hypopneas were finally used for our automatic hypopnea processing and analysis.

\section{E. Feature extraction}

As a result of the partial collapse of the UA during sleep, UA resistance increases, usually leading to inspiratory flow limitation (IFL). IFL has been formally defined as a minimum decrease of $1 \mathrm{cmH}_{2} \mathrm{O}(0.7356 \mathrm{mmHg})$ of esophageal (intrathoracic) pressure without a corresponding increase in airway flow rate $[8,9]$ and presents a characteristic inspiratory airflow pattern known as flattening $[8,9]$. Thus, the presence of IFL in form of flattening patterns in the airflow signal provides non-invasive information on changes in UA resistance and respiratory effort [8 - 11].

The incidence of IFL in inspiratory cycles is significantly higher in obstructive than in central hypopneas [10], so IFL incidence provides information on a hypopnea's etiology. We therefore implemented the automatic, non-invasive IFL classifier presented in [11] that uses time and spectral features extracted from the airflow signal to characterize the airflow pattern of each inspiration. When the automatic IFL classifier assessed an inspiration with IFL, it was assigned the value $\{+1\}$, while if it was a non-IFL inspiration it obtained the value $\{0\}$.

After this analysis, we sought after the features that could best characterize the differences between obstructive and central hypopneas. All these features, see Table I, were noninvasively obtained solely from the airflow signal. So, the mean IFL value for all inspirations of a hypopnea, see
TABLE I

FEATURES OF THE AUTOMATIC NON-INVASIVE CLASSIFIER

\begin{tabular}{|c|c|c|c|}
\hline \multicolumn{3}{|c|}{ Description } & Description \\
\hline 1 & $\begin{array}{l}\text { Mean IFL value of all the } \\
\text { inspirations in hflow }\end{array}$ & 7 & Quotient of feature 5 and 6 \\
\hline 2 & $\begin{array}{l}\text { Mean IFL value of all the } \\
\text { inspirations in flow } 2 \mathrm{~min}\end{array}$ & 8 & $\begin{array}{l}\text { Quotient of feature } 5 \text { and the } \\
\text { maximum duration in flow } 2 \mathrm{~min}\end{array}$ \\
\hline 3 & $\begin{array}{l}\text { Number of inspirations in } \\
\text { hflow }\end{array}$ & 9 & $\begin{array}{l}\text { Mean value of the maximas of all } \\
\text { inspirations in hflow }\end{array}$ \\
\hline 4 & $\begin{array}{l}\text { Number of inspirations in } \\
\text { flow } 2 \mathrm{~min}\end{array}$ & 10 & $\begin{array}{l}\text { Mean value of the maximas of all } \\
\text { inspirations in flow } 2 \mathrm{~min}\end{array}$ \\
\hline 5 & $\begin{array}{c}\text { Mean value of the } \\
\text { inspirations' duration in } \\
\text { hflow [s] }\end{array}$ & 11 & $\begin{array}{c}\text { Difference between feature } 8 \text { and } \\
\text { feature } 7\end{array}$ \\
\hline 6 & $\begin{array}{l}\text { Mean value of the } \\
\text { inspirations' duration in } \\
\text { flow } 2 \min [\mathrm{s}]\end{array}$ & 12 & $\begin{array}{l}\text { Maximal area of an inspiration in } \\
\text { hflow divided by the maximal } \\
\text { area of an inspiration in flow } 2 \mathrm{~min}\end{array}$ \\
\hline
\end{tabular}

feature 1 in Table I, and all inspirations of the $2 \mathrm{~min}$ prior to a hypopnea's onset, see feature 2 in Table I, were computed. Other features of airflow inspirations, such as its duration in time (seconds), see features $5-7$, or the area of the inspiration, see feature 12, were used for the characterization. A step-by-step outline of the automatic, non-invasive hypopnea classifier can be seen in fig. 2 .

To compare the performance of our automatic classifier, we used a manual algorithm [15] independently developed by researchers at our partner clinic. This non-invasive hypopnea classification algorithm for human experts allows human experts to manually differentiate hypopneas with classic NPSG signals, without using the Pes signal [15].

\section{$F$. Training and testing of the non-invasive classifier}

Discriminant Analysis (DA) is one of the techniques in supervised machine learning [16] with the lowest computational complexity and consequently shortest processing time. This classification method requires a training phase where the classifier learns to solve a multiclass problem. The training set usually consists of a (ntimes- $k$ ) matrix with a number $k$ of $n$-dimensional elements and of a label vector of length $k$ containing the class label $\{+1 ;-1\}$ for each element. In our case, the labels corresponded to the manual, gold-standard scorings for central $\{-1\}$ and obstructive $\{+1\}$ hypopneas.

Sequential forward feature selection was used to automatically select the most relevant subset of features for each classifier and achieve the best possible hypopnea classification results. Training, validation and test sets were created with a cross-validation algorithm in order to randomly assign $35 \%$ of the hypopneas to the training set, $15 \%$ to the validation set and $50 \%$ to the test set. Thus, the training set consisted of 321 hypopneas, the validation set of 138 hypopneas and the test set of 459 hypopneas The DA analysis was performed with 5 different functions: linear DA (LDA), diagonal linear DA (DLDA), quadratic DA (QDA), diagonal quadratic DA (DQDA) and Mahalanobis DA (MDA). 


\section{RESULTS}

The best classification results for DA were achieved with the diagonal quadratic DA (DQDA) function and a feature vector of length eight comprising the features $(1,4,5,6,7$, $8,11,12)$. The results of the automatic, non-invasive and the manual, non-invasive algorithm can be seen in Table II. While sensitivity and accuracy of both classifiers were approximately on par, the specificity of the manual classification was higher than that of the automatic classifier (Table II).

TABLE II

CLASSIFICATION RESULTS OF THE NON-INVASIVE CLASSIFIERS

\begin{tabular}{ccc}
\hline \hline & $\begin{array}{c}\text { Automatic } \\
\text { classification }\end{array}$ & $\begin{array}{c}\text { Manual } \\
\text { classification }\end{array}$ \\
\hline Sensitivity & 0.71 & 0.68 \\
Specificity & 0.67 & 0.85 \\
PPV & 0.76 & 0.87 \\
NPV & 0.61 & 0.65 \\
Accuracy & $\mathbf{0 . 6 9}$ & $\mathbf{0 . 7 5}$ \\
Features & $1,4,5,6,7,8,11,12$ & Flattening and \\
& $($ see Table I) & context-based \\
& & information
\end{tabular}

PPV: Positive predictive Value, NPV: negative predictive value Labels were defined as $\{+1\}$ for obstructive and $\{-1\}$ for central hypopneas.

\section{DISSCUSION AND CONCLUSIONS}

In this paper we have shown the feasibility of a new noninvasive approach for the automatic differentiation of central and obstructive hypopneas with nasal airflow. For this purpose we combined methodologies outlined in prior studies $[10,11]$. Unlike in a previous study [10], all features used here by our automatic classifier (Table I) for the hypopnea differentiation were exclusively extracted from the non-invasive airflow signal. IFL episodes were assessed by implementing the non-invasive IFL classifier suggested in [11].

To fairly compare our automatic algorithm's performance, we used a recently proposed manual hypopnea differentiation algorithm for human experts [15]. This algorithm also only employs features extracted from classic NPSG signals, while blinded to the Pes signal. For the goldstandard validation of this manual and our automatic noninvasive hypopnea classifiers, we employed the manual, gold-standard (Pes) hypopnea annotations. So, the validity of both differentiation scores should be ensured.

The results for our automatic hypopnea classifier seem promising, as the automatic classifier's accuracy is close to the accuracy obtained with the manual, non-invasive scoring, see Table II. Like most systems relying only on NPSG signals, the manual classification algorithm tended to over-classify central events $(0.85)$ in detriment of obstructive events (0.68), see Table II. The automatic classifier, however, showed a better balanced identification performance of the two hypopnea classes, see Table II. This underlines the importance of using objectively extracted respiratory effort related information (IFL/flattening) during the hypopnea's differentiation process. Furthermore, even though more than 200 additional hypopneas (approx. $+30 \%$ ) were analyzed in this study, the accuracy obtained here (0.69) was almost on par with the accuracy (0.72) obtained in a prior study [10] that used the gold-standard Pes/flow relationship to assess IFL. However, further development of the automatic, non-invasive differentiation system will be necessary to obtain a higher robustness, classification and generalization performance level compatible with clinical routine. Also a more extensive manual hypopnea annotation set scored by at least two independent human scorers will be necessary for a prospective clinical validation of the automatic algorithm.

Still, we are confident that the promising insights obtained in this study have shown the feasibility of an automatic, entirely non-invasive hypopnea differentiation system solely based upon the airflow signal.

\section{REFERENCES}

[1] Pack AI, Advances in Sleep Disordered Breathing, Am J Respir. Crit Care Med 172, 7-15, 2006

[2] The Report of an American Academy of Sleep Medicine Task Force, Sleep-Related Breathing Disorders in Adults: Recommendations for Syndrome Definition and Measurement Techniques in Clinical Research, SLEEP, Vol. 22, No. 5, 1999

[3] Iber, C., Anoni-Israel S., Chesson A. L., Qua S.F., The AASM Manual for the Scoring of Sleep and Associated Events, American Academy of Sleep Medicine, Westchester, IL, 2007

[4] O. Fontenla-Romero, B. Guijarro-Berdiñas, A. Alonso-Betanzos, V. Moret-Bonillo, A new method for sleep apnea classification using wavelets and feedforward neural networks, Artificial Intelligence in Medicine, 34:1, pp. 65-76, 2005

[5] Steltner H, Staats R, Timmer J, Vogel M, Guttmann J, Matthys H, Virchow JC, Diagnosis of Sleep Apnea by Automatic Analysis of Nasal Pressure and Forced Oscillation Impedance, Am J Respir Crit Care Med Vol 165. pp 940-944, 2002

[6] Yen FC, Behbehani K, Lucas EA, Burk JR, Axe JR, A Nonnvasive Technique for Detecting Obstructive and Central Sleep Apnea, IEEE Transactions on Biomedical Engineering, Vol. 44, No. 12, 1997

[7] Argod J, Pépin JL, Lévy P., Differentiating Obstructive and Central Sleep Respiratory Events through Pulse Transit Time, Am J Respirat Crit Care Med 158:1778-1783. 1998

[8] Hosselet J., Norman R., Ayappa A.,Rapoport D., Detection of Flow Limitation with a Nasal Cannula/ Pressure Transducer System, Am J Respir Crit Care Med Vol 157. pp 1461-1467, 1998

[9] Clark S. A., Wilson C. R., Sato M., Pegelow D., Dempsey J. A., Assessment of Inspiratory Flow Limitation Invasively and Noninvasively during Sleep, Am J Resp Crit Med, 158, 713-722, 1998

[10] Morgenstern C., Schwaibold M., Randerath W., Bolz A., Jané R., An invasive and a non-invasive approach for the automatic differentiation of obstructive and central hypopneas , IEEE Trans. Biomed. Eng., in press, DOI: 10.1109/TBME.2010.2047505, 2010

[11] Morgenstern C., Schwaibold M., Randerath W., Bolz A., Jané R., Assessment of changes in upper airway obstruction by automatic identification of inspiratory flow limitation during sleep, IEEE Trans. Biomed. Eng., Vol. 56 (8), pp. 2006-2015, 2009

[12] Benditt, JO, Esophageal and Gastric Pressure Measurements, Respir Care, 50(1):68 -75, 2005

[13] Varady P, Micsik T, Benedek S, Benyo Z. A novel method for the detection of apnea and hypopnea events in respiration signals. IEEE Trans. Biomed Eng 49(9):936-942, 2002

[14] T. Aittokallio, O. Nevalainen, U. Pursiheimo, T. Saaresranta, O. Polo, Classification of Nasal Inspiratory Flow Shapes by Attributed Finite Automata, Computers and Biomedical Research 32, 34-55, 1999

[15] W. Randerath, C. Morgenstern, A. Oltmann, C. Priegnitz, M. Treml, N. Anduleit, Evaluation of a new PSG based algorithm to differentiate obstructive and central hypopnoeas, presented at the $20^{\text {th }}$ Annual Conf. of the European Respiratory Society, Barcelona, Spain, 2010

[16] Krzanowski, W. J., Principles of Multivariate Analysis, Oxford University Press, 1988 